\title{
Peri implantitis: An overview
}

\author{
Benita Maria Regi ${ }^{*}$, S Savita ${ }^{2}$, Gautami Kaimal ${ }^{3}$ \\ ${ }^{1,3}$ Postgraduate, ${ }^{2}$ Professor and HOD, Dept. of Periodontology, Rajarajeswari Dental College and Hospital, Bangalore, Karnataka, India \\ *Correspondig Author: Benita Maria Regi ${ }^{1}$ \\ Email: benitaregi@gmail.com
}

\begin{abstract}
Biologic complications associated with dental implants primarily relate to infection in the soft and hard tissue around implants known as peri implant mucositis and peri implantitis respectively. Prevalence of peri implantitis rates upto $60 \%$ and lead to the loss of implants. Current evidence points to poor oral hygiene, history of periodontitis, and smoking as being the strongest risk indicators for peri implantitis along with the factors affecting plaque accumulation and removal like design of prosthesis and excess cement. Different methods are used to assess peri implant tissue health and to diagnose these entities. Various treatment modalities are available including conservative and surgical approaches for the treatment of peri implant diseases so as to achieve reosseointegration of the exposed implant surface, being the ultimate goal. The aim of this review is to provide an overview regarding etiology, diagnosis and treatment of peri implantitis.
\end{abstract}

Keywords: Mouthwash, Chlorhexidine, Stains, Bacteria.

\section{Introduction}

The introduction of dental implants has created a paradigm shift in the orodental rehabilitation of patients. They are proven and well-established treatment modality which helps to restore esthetics and compromised oral function resulted from tooth loss. Evidences proved the safe use of dental implants and has been presented since 1960s and 1970s. Despite the high success and survival rates of oral implants, failures do occur and implant-supported prosthesis may require a substantial periodontal and prosthodontic maintenance over time. ${ }^{1}$ Peri-implant tissues are more liable to inflammatory disease than periodontal tissues due to diminished vascularization and parallel orientation of the collagen fibres. This phenomenon can be verified immunohistochemically through increased formation of inflammatory infiltrate, in comparison with the teeth. ${ }^{2}$

\section{Definition}

According to American Academy of Periodontology, periimplantitis is defined as an inflammatory process around an implant, including both soft tissue inflammation and progressive loss of supporting bone beyond biological bone remodeling.

\section{Epidemiology}

Peri-implantitis has been known to affect $28-56 \%$ of the subjects and $12-43 \%$ of the implants, although epidemiological data are limited. ${ }^{3}$ Based on the 6th European Workshop on Periodontology consensus report, Lindhe \& Meyle, reported peri implantitis rate between $28 \%$ to $58 \% .^{3}$

\section{Etiology}

\section{Subgingival microbiology and dental implants}

In good oral health, microflora with streptococci and nonmobile rods predominate, in both teeth and implants. The same groups of periodontopathogens are recognized in periodontal diseases and peri implantitis. Commonly found microflora are A. actinomycetemcomitans, P. gingivalis, T. forsythia, P. intermedia, C. rectus. There are five lines of evidence that support the role of microorganisms in causing peri-implantitis. ${ }^{4}$

1. Human experiments, reveals plaque deposition on implants, that can induce peri-implant mucositis,

2. Distinct quantitative and qualitative differences are demonstrated in the microflora associated with successful and failing implants,

3. Shift in the composition of the microflora and periimplantitis due to placement of plaque - retentive ligatures in animals,

4. Clinical status of peri-implantitis patients improved by antimicrobial therapy and,

5. Evidence indicates that oral hygiene level has an impact on the long-term success of implant therapy.

\section{Biomechanical overload}

Excessive biomechanical forces/ or overloading may lead to high stress or microfractures at the coronal aspect of implant bone interface, thereby causing bone loss. The apical downgrowth of epithelium and connective tissue result in loss of osseointegration around the implant region. The degree of loss of implant bone contact depends on the frequency and magnitude of the occlusal loading as well as superimposed bacterial invasion. Naert et al (1991), reported greater bone loss around the implant with respect to the magnitude of implant loading. ${ }^{5}$

\section{Other etiologic factors \\ Patient related factors}

1. Systemic diseases like diabetes

2. Smoking

3. Poor plaque control/irregular maintenance therapy

4. Para functional habits

5. Inadequate amount of bone resulting in an exposed implant surface at the time of placement 


\section{Iatrogenic factors}

1. Traumatic surgical techniques

2. Lack of primary stability

3. Premature loading during the healing period

\section{Classification}

1. Jovanovic \& Klinge 1990, Spiekermann 1991 classified on the basis of clinical status of peri-implant bone during various stages of peri implantitis and required therapy

Class I: - Slight horizontal bone loss with minimal periimplant defect

Class II: - Moderate horizontal bone loss with isolated vertical defect

Class III: - Moderate to advanced horizontal bone loss with broad, circular bony defect

Class IV: - Advanced horizontal bone loss with broad, circumferential vertical as well as loss of the oral or vestibular bony wall

2. Newman 1992: Based on severity of peri-implantitis and sub classification of non-successful implants.

a. Compromised successful implant: Inflammation, hyperplasia, and fistula formation occur near an otherwise fully osseointegrated implant.

b. Failing implant: The implant is characterized by progressive bone resorption, but remains functional.

c. Failed implant: Infection persist around an implant whose function is compromised

d. Other classifications

e. Froum \& Rosen 2012

f. Ata Ali et al, 2015

g. Renvert \& Claffey, 2013

h. American Academy of Periodontology 2013

i. Carl E Misch \& Jon B Suzuki 2014

\section{Clinical features}

a. Presence of an inflammatory lesion in the mucosa around the implants

b. Peri-implant probing depth

c. Bleeding on probing

d. Exudation \& suppuration

e. Pain - not a typical feature

f. Hyperplasia and swelling of the tissues around implants.

\section{Diagnosis}

\section{A) Peri implant probing depth}

Assessment of peri-implant probing depth, bleeding on probing, exudation and suppuration from the peri-implant space is performed with blunt, straight periodontal probe. Plastic probes avoid damage and contamination of implant titanium surface. Pockets 5 or more $\mathrm{mm}$ deep may be a protected habitat for putative pathogens and is a sign of peri-implantitis. ${ }^{4}$ Lang et al. reported that in healthy and mucositis sites, the probe tip was at the most apical cell of the junctional epithelium, whereas in case of ligatureinduced peri-implantitis sites the probe tip penetrated into the connective tissue. They concluded that peri-implant mucosal health or disease status can be assessed by probing around implants. Usually large probing depths are often the first clinical sign of an insidious infectious process in the peri implant tissues. ${ }^{6}$

\section{B) Bleeding on probing (BOP)}

It is recommended that bleeding on probing, to be encompassed in routine evaluation of dental implants as it indicates the presence of inflammation around the implant mucosa and can be a predictor for future loss of tissue support. A light standardized probing force $(0.2-0.25 \mathrm{~N})$, was recommended to monitor periodontal and peri-implant status. Bleeding on probing in Beagle dogs was detected in $0 \%$ of healthy sites, in $67 \%$ of mucositis sites and in $91 \%$ of peri-implantitis sites. Assessment of bleeding on probing and microbiological testing in sites with progressive periimplantitis, concluded that 'bleeding on probing is a useful clinical parameter for predicting both periodontal and periimplant attachment loss. ${ }^{6}$ Some authors reported that BOP indicates inflammation around implants whereas other studies infer that it may occur even in the presence of healthy peri implant soft tissues (Quirynen et al 1992).

\section{C) Mobility}

Implant mobility is suggestive of terminal stage of peri implant disease, characterized by complete loss of bone to implant interface and imply lack of osseointegration. This parameter diagnoses the final stage of osseo disintegration and may help to decide that an implant has to be removed. For the interpretation of low degrees of mobility, an electronic device has been proposed, which was originally designed to measure the damping characteristics of the periodontium of natural teeth (Periotest). ${ }^{4}$

\section{D) Peri-implant radiography}

The primary success criterion for implant systems is the preservation of marginal bone height. Standardized IOPA radiographs and orthopantomogram are used. Vertical bone loss, saucer shaped defect and progressive bone loss suggest peri-implantitis. Usually saucer shaped defects are seen around the implant (certain cases, wedge shape defects) while the bottom part of the implant maintains perfect osseointegration. Until osseo-integration is lost, destruction of bone may progress without any signs of implant mobility clinically. A continuous periimplant radiolucency indicates implant failure. Vertical bone loss of $<0.2 \mathrm{~mm} /$ year has been proposed as one of the major criteria for success.

\section{E) Peri implant sulcular fluid analysis and periotron}

In 1991, Tetsch recommended the measurement of sulcular fluid flow rate (SFFR) as a technique to quantitatively evaluate peri implant inflammation. Correlations between probing depths and SFFR values exist (Sporlein et al1986, Gunay et al 1990). Filter paper strips that have been used to collect fluid from sulcus, that are inserted into periotron for the measurement of flow rate. An increase in gingival sulcus fluid is generally accepted as a sign of marginal peri implant inflammation. In 1991, Tetsch proposed a classification of these periotron values: $<10$ - absolutely inflammation free 
gingiva, $10-40$ - mild inflammation, >40 - acute peri implant infections.

\section{F) Microbial monitoring}

This is to determine the microbial configuration of periimplantitis site. Various techniques to monitor subgingival flora for determining an elevated risk for periodontal disease or peri-implantitis includes bacterial culture, DNA probes, monoclonal antibody, enzyme assays and polymerase chain reaction. At present, the benefit of microbiological tests as a primary tool in determining the risk for peri-implant tissue loss cannot be explicited. ${ }^{4}$

\section{G) Osstell}

A device developed by Huang to evaluate the implant bone interface which is non-destructive and non-invasive. It is based on resonance frequency analysis. ${ }^{7}$

\section{Suggested risk-assessment parameters}

In practical terms, a low-risk-profile patient is one who has responded favourably to periodontal therapy and presents with optimal oral hygiene, does not smoke, is systemically healthy and runs a low risk for periodontal disease. In such a patient, the risk associated with replacement of lost / hopeless / questionable teeth with dental implants will be low.

A patient with a moderate-risk profile has, limited number of residual sites with probing pocket depth $\geq 5 \mathrm{~mm}$ that bleed upon probing following completion of periodontal therapy and the oral hygiene is not constantly optimal. Before any final restorative treatment plan with dental implants, an attempt for further pocket reduction should be considered. At this stage, the dentist should consider restorative treatment options other than dental implants, such as interim tooth-supported reconstructions.

A patient with a high-risk profile has a significant number of residual sites with probing pocket depths $\geq 5 \mathrm{~mm}$ that bleed upon probing, the oral hygiene is suboptimal and/ or the patient is a heavy smoker and / or, for example, suffers from uncontrolled type 2 diabetes. Implant placement should be delayed in such patients and should provide periodontal treatment, as maintenance of teeth should be the priority. ${ }^{8}$

\section{Treatment plan}

\section{A) Non-surgical therapy}

Non-surgical therapy aims at infection control through debridement of the implant surface and thereby minimizing the bacterial load below the threshold level for causing disease. $^{3}$

\section{Occlusal therapy}

When there are excessive forces, prosthesis design changes, improvement in implant number and position occlusal adjustment should be done.

\section{Debridement}

Debridement systems include curettes and ultrasonic devices.

\section{IIa) Curettes}

Curettes made of numerous materials are used to debride implant surfaces. Carbon-fiber curettes eliminate bacterial deposits without disrupting the surface of the implant, as they may be softer than the implant surface but wreck easily. Teflon curettes are used as an adjunct to air-abrasive systems. Plastic curettes are brittle among all curette types. Titanium-coated curettes have comparable hardness to the titanium surface and thus do not roughen its surface while steel curettes have external hardness higher than titanium and no longer indicated for titanium implants. Though, they can be used on other implant surfaces including titanium zircon oxide or titanium oxynitride. ${ }^{3}$

\section{IIb). Ultrasonic devices}

The ultrasonic devices eliminate biofilm and calculus without altering the implant surface. To accomplish this, refinement of tips has been used including carbon fiber, silicone or plastic. Another modification to the conventional ultrasonic device is the Vector system, in which the horizontal vibration is transformed to a vertical vibration with the aid a resonating ring, ensuing in a parallel motion of the running/working tip to the surface. Ultrasonic devices with polyether ether ketone-coated tips can also be used to debride the implant surface.

\section{Air abrasive systems}

Standard powdered air-abrasive systems use the sodium bicarbonate containing air-spray. They eliminate tooth stains and can be used for polishing, but cannot be used for implant instrumentation because they may deteriorate hard and soft tissue due to their excessive abrasiveness. Latterly, a powered air abrasive system, based on a low-abrasive amino-acid glycine powder, has been advocated for debriding implant surfaces and is illustrated as an effective method of biofilm removal, without damaging hard and soft tissues.

\section{Adjunctive antimicrobial products and antiseptics}

Antiseptics and antibiotics, have been used adjunctively to enhance the effects of nonsurgical debridement, thereby minimizing bacterial loads. Chlorhexidine-based products can be used as gels, irrigation and/or rinses, and in different formulations and regimes. Examples encompasses irrigation of the peri-implant pocket with $0.2 \%$ chlorhexidine in a single session, use of $1 \%$ chlorhexidine gel in a single or repeated application at baseline treatment and at 30 and 90 days post treatment, pocket irrigation with $0.12 \%$ chlorhexidine plus $1 \%$ chlorhexidine gel. ${ }^{3}$ There are numerous types of antiseptics available including $\mathrm{NaOCl}$ $1.0 \%, \mathrm{H} 2 \mathrm{O} 23.0 \%, 0.12 \%$ or $0.2 \%$ of chlorhexidine gluconate, citric acid $40.0 \%$, EDTA $24 \%$, povidone iodine $10 \%$, phenols and essential oils. ${ }^{7}$

\section{Citric acid}

Followed with the aid of debridement of the surface of implant, a cotton pellet with a smidgen of $40 \%$ citric acid is 
burnished onto exposed implant surfaces for 30 to 60 seconds. Biofilm penetration, long substantivity, tissue biocompatibility, and low resistance are the properties of citric acid. ${ }^{7}$

\section{Locally or systemically delivered antimicrobials}

Various locally or systemically delivered antimicrobials includes $1 \mathrm{mg}$ minocycline and $3 \mathrm{mg}$ of poly(glycolide-codl-lactide) placed submucosally at each treatment site 30 and 90 days after treatment or topical irrigation with a solution containing $8.5 \%$ by weight of doxycycline and $37 \%$ by weight of poly-DL-lactide dissolved in a biocompatible carrier of N-methyl-2-pyrrolidone.

A study revealed reosseointegration after 4 months with the application of pure tetracycline. It is exceptionally endorsed to comprise tetracycline in periimplantitis treatment. Systemic antibiotics are also administered adjunctively to mechanical debridement in order to attain effective antimicrobial levels in the peri-implant crevicular fluid and therefore to aid the antibacterial mechanical effect. Many researches established amoxicillin and metronidazole aggregate as the most effective antimicrobials. For patients who are allergic to amoxicillin, doxycycline $10 \%$ controlled release gel such as Atridox may also be taken into consideration. ${ }^{?}$

\section{Laser decontamination and photodynamic therapy}

Using lasers has been broadly frequent in the treatment of peri-implantitis due to their anti-infective, physical and ablation properties. CO2, Diode-, Er: YAG- (erbium-doped: yttrium-aluminium-garnet) and Er, Cr: YSGG- (erbium, chromium-doped: yttrium scandium-gallium-garnet) lasers are used as a treatment modality in the management of periimplant diseases. Er: YAG laser is widely accepted due to its potential to eliminate subgingival plaque and calculus without damaging the implant surface. Treatment with a CO2 $308 \mathrm{~nm}$ excimer laser, has also shown satisfactory results in an anaerobic bacterial spectrum. ${ }^{3}$ A companion protocol which is laser assisted peri implantitis protocol, used to address mild to moderate peri-implantitis. It is generally performed under local anesthesia and it requires 1 or 2 appointments of $2 \mathrm{hrs}$ each. ${ }^{7}$ Photodynamic therapy should also be considered as an additional treatment option. ${ }^{2}$

\section{B) Surgical therapy in peri implantitis}

The predominant goal of surgical treatment of periimplantitis is to provide implant surface cleanability, thereby changing the anatomy of soft and hard peri-implant tissues in order to acquire re-osseointegration.

\section{Surface polishing / implantoplasty (Before resection)}

This method pursuits for implant surface smoothening, thereby altering the rough implant surface to a polished surface which is amenable for oral hygiene maintenance. Implant topography is altered using high-speed diamond burs and polishers which creates smooth continuous surfaces. This technique is performed before any osseous resective therapy and is used with profuse irrigation.
Various surgical techniques are recommended:3,7

1. access for cleaning and decontamination of the implant surface (access flaps);

2. access for cleaning and decontamination plus exposure of the affected surfaces for cleaning (apically repositioned flaps); and

3. access for cleaning plus aiming for bone regeneration and re-osseointegration (regenerative techniques). ${ }^{3,7}$

\section{Cumulative interceptive supportive therapy (CIST) Protocol}

In the course of maintenance, developing periimplant lesions can be treated with Cumulative Interceptive Supportive Therapy (CIST) protocols (Fig. 1). It includes mechanical, antiseptic and antibiotic treatment to control ongoing infection. Following this, periimplant bony lesions could also be corrected by regenerative or resective surgical techniques. It is evident that preventive measures need to be reinstituted following such therapy. ${ }^{9}$

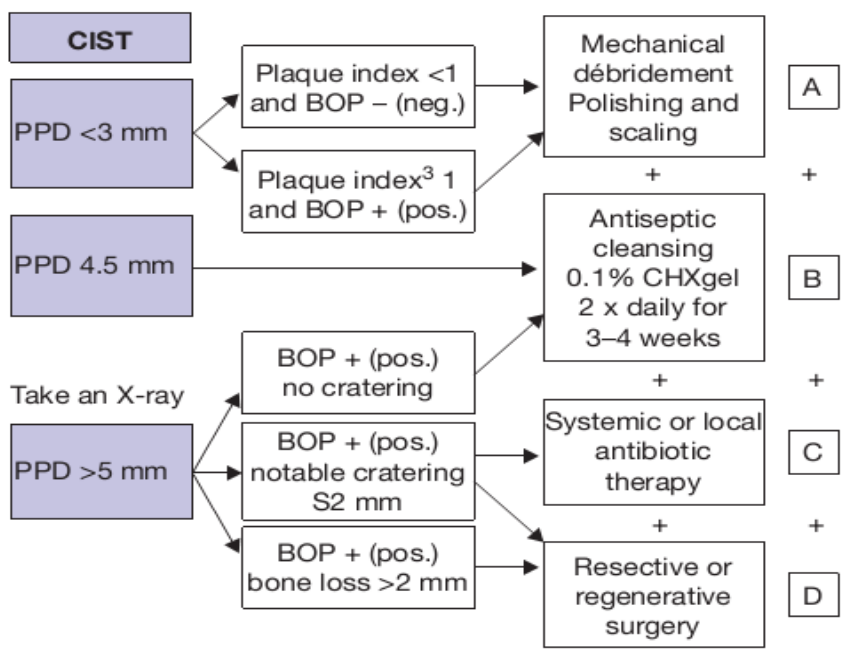

Fig. 1: CIST Protocol

\section{Maintenance}

After surgical intervention, all patients have to be recalled in every 3 months as maintenance visits for monitoring plaque levels, soft tissue inflammation, and changes within the bone level.

\section{Conclusion}

Patient should be aware about the chances of developing inflammation and infection around implants. A supportive, preventative program based on a risk assessment is mandatory for all patients. Therefore, dentists should acknowledge and inform implant patients, the unquantifiable risk of peri-implantitis and maintenance visits for monitoring oral hygiene, soft tissue inflammation, and changes happening in the bone.

\section{Source of funding}

None. 


\section{Conflict of interest}

None.

\section{References}

1. Esposito M, Hirsch JM, Lekholm U, Thomsen P. Biological factors contributing to failures of Osseo integrated implants Success criteria and epidemiology. Eur J Oral Sci 1998;106:527-55.

2. Smeets R, Henningsen A, Jung O, Heiland M, Hammaches C, Stern JM. Definition, etiology, prevention and treatment of peri implantitis - a review. Head Face Med 2014;10:34.

3. Figuero E, Graziani F, Sanz I, Herrera D, Sanz M.

Management of peri-implant mucositis and peri-implantitis. Periodontol 2000 2014;66(1):255-73.

4. Mombelli A, Lang NP. The diagnosis and treatment of periimplantitis. Periodontol 2000 1998;17:63-76.

5. Schwarz F, Derks J, Monje A, Wang HL. Peri-implantitis. $J$ Periodontol 2018;89(Suppl 1):S267-S90.

6. Coli P, Christiaens V, Sennerby L, Bruyn HD. Reliability of periodontal diagnostic tools for monitoring peri-implant health and disease. Periodontol 2000 2017;73:203-17.
7. Suzuki BJ, Carl E Misch. Periodontal and maintenance complications. Textbook of avoiding complications in oral implantology. 2018:771-826.

8. Donos N, Laurell L, Mardas N. Hierarchical decisions on teeth vs. implants in the periodontitis susceptible patient: The modern dilemma. Periodontol 2000 2012;59:89-110.

9. Lang NP, Berglundh T, Heitz Mayfield LJ, Pjetursson BE, Salvi GE, Sanz M. Consensus statements and recommended clinical procedures regrading implant survival and complications. Int J Oral Maxillofac Implants 2004;19(Suppl):150-4.

How to cite: Regi BM, Savita S, Kaimal G. Peri implantitis: An overview. IP Int J Periodontol Implantol 2020;5(1):11-5. 